C2004 IEEE. Personal use of this material is permitted. However, permission to reprint/republish this material for advertising or promotional purposes or for creating new collective works for resale or redistribution to servers or lists, or to reuse any copyrighted component of this work in other works must be obtained from the IEEE. 


\title{
Quantum Computing With Superconductors
}

KARL K. BERGGREN, MEMBER, IEEE

\author{
Invited Paper
}

Superconductive technology is one of the most promising approaches to quantum computing because it offers devices with little dissipation, ultrasensitive magnetometers, and electrometers for state readout, large-scale-integration, and a family of classical electronics that could be used for quantum bit (qubit) control. The challenges this technology faces, however, are substantial: for example, control of the qubit to a part in $\sim 10^{4}$ must be accomplished with analog control pulses. But even after this is done, the accuracy is limited by the unavoidable decay of quantum information in the system. Recent experiments suggest the time over which this decay occurs is $<1 \mu \mathrm{s}$, though it is expected to lengthen as experimental methods improve. A 1- $\mu$ s decay time would mandate a very difficult to achieve maximum time of $\sim 100$ ps per analog operation. Thus, quantum computing is, simultaneously a promising technology for solving certain very hard problems in computer science and a daunting challenge for those working to develop that technology.

Keywords-Josephson junction qubit, macroscopic quantum coherence, quantum computing, superconductive qubit.

\section{BACKGROUND}

In 1994, the discovery by Shor, then at AT\&T Bell Laboratories, of a method of factoring large numbers efficiently using quantum computers [1], transformed quantum computing from a fascinating academic curiosity to a major cross-disciplinary field of basic research. Because the difficulty of factoring large numbers (or, more precisely, the challenge of solving the discrete logarithm problem) underlies the security of ubiquitous public-key cryptography methods [2], Shor's invention immediately sparked a huge experimental effort to develop a practical quantum computer, large enough to be of use for such an application. Approaches based on trapped ions, neutral atoms, photons, nuclear magnetic resonance, and a variety of solid state systems have been investigated in recent years [3], [4]. Despite

Manuscript received December 6, 2003; revised March 22, 2004. This work was supported in part by the Air Force Office of Scientific Research under Air Force Contract F19628-00-C-002.

The author was with MIT Lincoln Laboratory, Lexington, MA 02420 USA. He is now with the Department of Electrical Engineering and Computer Science, Massachusetts Institute of Technology, Cambridge, MA 02139 USA (e-mail: berggren@mit.edu).

Digital Object Identifier 10.1109/JPROC.2004.833672 great progress, quantum computers large enough to factor a useful number remain many years off.

Superconductive technology is a particularly attractive technology for implementing quantum computing circuits for two reasons: 1) the physics of superconductivity provides a quantum system that can be engineered using microfabrication methods to have a range of desirable properties and 2) superconductive circuits can be integrated monolithically and then scaled in complexity [5]-[8]. As we will now discuss, this scaling is critical, because quantum computers need to be quite large and sophisticated before they can be applied to real-world applications, and many of the alternative technologies (such as atom- or ion-based methods, and liquid-state nuclear magnetic resonance (NMR)] are not scalable by conventional methods of integrated-microelectronic-circuit manufacturing.

To illustrate the importance of scalability to quantum computing, Fig. 1 compares an estimate of the time required to factor numbers of various lengths using either a quantum computer, or a hypothetical classical computer roughly 30 times faster than the fastest supercomputer that now exists. Scaling up from demonstrations of classical factoring of smaller numbers [9], we can estimate that a 2048-b-long number would require execution time on the classical computer longer than the age of the universe, while realistic estimates of what might be achievable in a quantum computer running with a clock speed of only $100 \mathrm{MHz}$ indicate that it could factor the same number in one hour [10]. This $100-\mathrm{MHz}$ computer would have to be large, however: straightforward scaling of estimates given in [10] and [11] suggest that $\sim 4 \times 10^{6}$ qubits (a qubit is a two-state quantum memory register) would be required to factor a 2048-b key. This scale is far beyond that of the few-qubit quantum computers that have been realized thus far [12].

Explaining in detail the reason quantum computers might be expected to operate faster than classical computers is beyond the scope of this article (see reviews such as [3], [4]); however, a simple analogy can be drawn with parallel computation that helps give a sense of the origin of the speedup.

In all computers, information is encoded at its most basic level in the state of a physical system. For example, in con- 

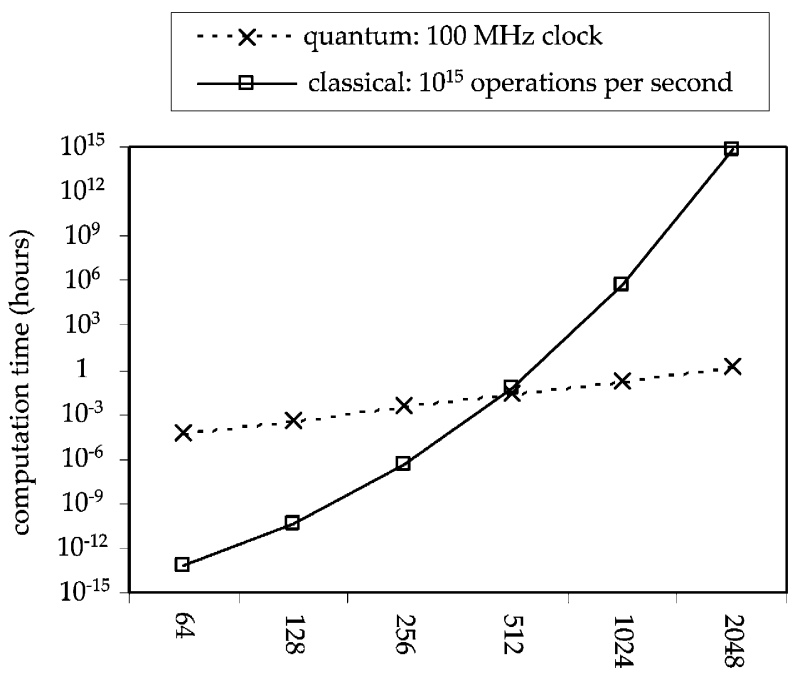

binary length of number to be factored

Fig. 1. Comparison of estimated computation time in hours for the problem of factoring numbers of various bit lengths for a quantum computer operated at $100-\mathrm{MHz}$ clock speed, and a classical computer capable of $10^{15}$ operations/s. The times shown span from picoseconds to many times the age of the universe. The quantum computer shows a clear advantage in scaling with binary-length of the key. The values shown were derived by scaling of the calculations from [9] and [10].

ventional silicon-based computers, the amount of charge on a capacitor encodes the logical value of a bit. But quantum mechanics tells us that physical systems can exist in more than one state simultaneously. These "superpositions" of states are completely outside of our everyday physical intuition, for two reasons: 1) they only exist while the physical system is totally isolated-an uncommon situation-and 2) their physical manifestations in large objects are evident only at extremely small time and length scales. But many carefully executed experiments, such as matter-interference experiments, have now proven that this description is indeed accurate for microscopic systems [13]-[15]. Indeed, superconductive quantum computation relies on quantum states that involve large numbers of particles (microamperes of current are not uncommon), and so this work is partly of interest because it has the potential to increase the size scale at which the more bizarre properties of quantum mechanics, such as superposition, are known to apply [16].

A quantum computer achieves a speedup over conventional computers by exploiting superposition to perform many calculations in parallel. Rather than operating on a single value of a memory register, as in a classical computer, the quantum computer can operate on all possible values of the register at once. Unfortunately, the delicate superposition state is destroyed when a measurement is made of the state of the computer (i.e., when output is requested from the computer). Still, the consequences of the superposition (the interference that results from the superposition) can encode the answer to a problem. This picture implies that a speed-up is only expected for problems where large memory spaces are required internal to the computer, but where the input and output registers can be small. Factoring is one example of such an algorithm - one of the very few algorithms where a speedup has been theoretically demonstrated.

The other main algorithm for quantum computing where a speedup over classical operation has been demonstrated is the so-called "database-search" algorithm proposed by Grover [17]. Although this algorithm could not, in fact, be used to accelerate repeated searches of an arbitrary database because the database itself is destroyed after each search-the correct analogy is to a telephone book you have to burn and rewrite every time you use-it may have other applications. For example, Brassard pointed out that if the database were generated internally to the algorithm itself, Grover's algorithm could be used to accelerate a particular type of code breaking operation [18].

There are other algorithms in development that could potentially use a quantum computer to perform important tasks that cannot currently be accomplished efficiently by a classical computer. Quantum lattice gas automata have been proposed, for example, that take advantage of a close analogy between the classical equations of motion of fluid flow and Schrödinger's equation to perform simple simulations of classical fluid flow on an array of small quantum computers, interfaced by classical communication channels [19]-[26]. Another proposed algorithm solves certain difficult problems using the fact that adiabatic evolution of a system will keep it in its ground state [27]-[30]. The system's potential is varied adiabatically in time such that any nonoptimal solution presents an energy cost to the final potential energy. Attempts have also been made to develop algorithms for image processing on quantum computers [31], [32]. While these suggestions are architecturally interesting, particularly because they purport to be able to harness useful computation with only a handful of qubits, the extent to which they scale to larger, more interesting problems has not yet been established theoretically.

\section{TeChnological Considerations}

Architectures that promise greater performance with fewer qubits are of particular interest for quantum computing because of the daunting engineering challenges posed in increasing the complexity of even nominally "scalable" approaches to quantum computing, like superconductive circuits. These challenges come about in part because quantum mechanics imposes apparently contradictory constraints on the devices: the devices must exhibit no power dissipation and must in all other ways be decoupled from their environment; but, at the same time, the devices must store a quantum signal that can be measured by an apparatus that is strongly coupled to the outside world. Typically, these requirements mean operating with nanometer-scale systems (such as single atoms, or nanometer-scale electronics), but superconductive circuits can meet these constraints with devices of much larger length scales because of their unique physics.

1) At frequencies well below the superconducting gap, superconductive circuits are nearly lossless. 


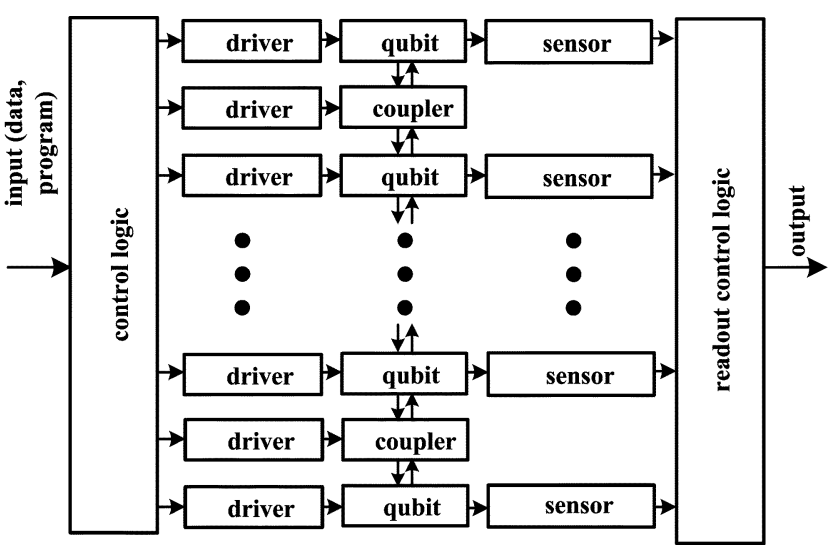

Fig. 2. Example architecture of superconductive quantum computer, indicating the main components: control logic to handle input and output, drivers that control single qubits and turn on and off interqubit interactions, and sensors to detect the qubit state at the end of the computation.

2) The Josephson junction, a nonlinear superconductive circuit element based on coherent charge-carrier (Cooper pair) tunneling, can be used to switch the coupling between various parts of the circuits.

3) Superconductive circuits can be integrated using standard microfabrication methods [33], [34].

4) Ultrasensitive magnetometers [35], [36] and electrometers [37] can be fabricated out of superconductors to read out the state of the computer.

5) A classical control electronics family (single-flux quantum (SFQ) electronics) [38], [39] exists that can be integrated with the superconductive qubits for classical control operations [40]-[42].

To examine in more detail the requirements for a technology, it is helpful to have a sample architecture in mind. The one illustrated in Fig. $2^{1}$ should not be considered as optimum in any sense. It is a simple linear array of qubits, interacting only with its nearest neighbors. Still, it shares the key features of more realistic architectures, and so it is useful for analysis.

\section{A. The Qubit}

The most important element of any quantum computing architecture is the qubit, a two-level quantum system that serves as a memory register for the computer. The computational basis, physical states that correspond to the logical values of one and zero, can be assigned arbitrarily to these two states or to any orthogonal linear superposition of these states. Fig. 3 shows the potential energy of an example quantum system-a simple $L-C$ oscillator. Expressed as a function of the magnetic flux $\boldsymbol{\Phi}$ in the inductor, the Hamiltonian that governs the behavior of this circuit is

$$
\begin{aligned}
H & =\frac{1}{2} C V^{2}+\frac{1}{2} L I^{2} \\
& =\frac{1}{2} \tilde{m} \dot{\Phi}^{2}+\frac{1}{2} \tilde{k} \Phi^{2}
\end{aligned}
$$

\footnotetext{
${ }^{1}$ This architecture was first suggested to me by Prof. J. E. Mooij.
}

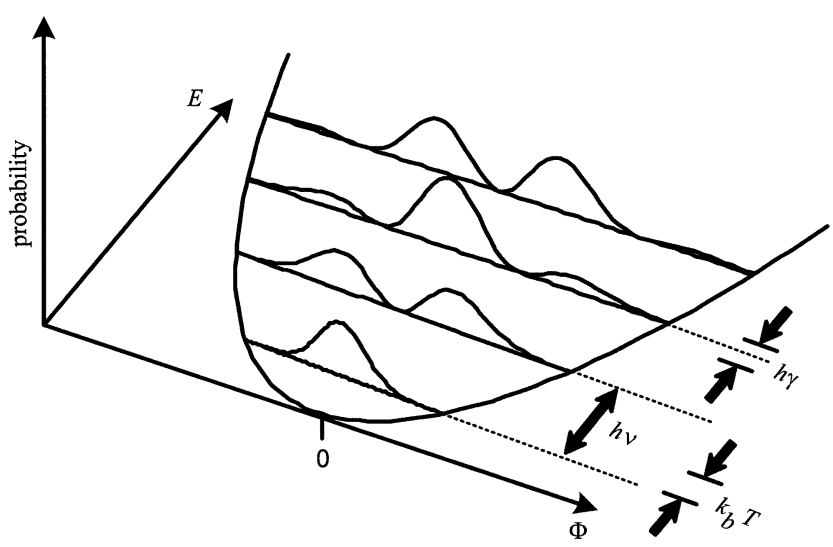

Fig. 3. Drawing of quantized states of the potential-energy diagram of an ideal $L-C$ oscillator. The $x$ axis in this diagram corresponds to the magnetic flux stored in the inductor, so the quantum eigenstates represent distributions of current. $k_{b}$ is Boltzmann's constant.

where $\tilde{m}$ is an effective mass (proportional to the capacitance) and $\tilde{k}$ is an effective spring constant (inversely proportional to the inductance). This expression is also the Hamiltonian of a simple harmonic oscillator, whose quantum states are analytically solvable and are shown in Fig. 3. For the circuit's quantum-mechanical nature to be important in describing its behavior, two key conditions must be met.

1) The circuit must be underdamped, so the linewidth of the states $\gamma$ is much less than the spacing between the states $h \nu$. This condition is readily satisfied in a superconductive circuit.

2) The resonance frequency must be high enough, or the temperature $T$ must be low enough, so that only the ground state is occupied. This constraint mandates temperatures of operation $\sim 10 \mathrm{mK}$, which can be achieved in a dilution refrigerator.

Because the harmonic oscillator energy states are evenly spaced, resonant electromagnetic excitation leads to multiphoton processes that occupy excited states, and thus do not provide the clean two-level system that is needed for quantum computing. Instead a nonlinear element, like the Josephson junction, must be introduced. These circuit elements produce anharmonic oscillators, so the quantum states have varying energy-level spacings and two-level systems can be conveniently manipulated in isolation.

The quantum behavior observed in a qubit is very different from the "quantum" in the term SFQ electronics (the classical superconductive electronics family). "Quantum" in SFQ refers to quantization of the flux in a superconductive loop. In an SFQ circuit the system is described by a well-defined current and voltage which evolves in time, while the state of a qubit is described by a distribution of currents or voltages, each occurring with some amplitude and phase, which evolve in time.

Several different styles of qubits have been implemented in superconductors, all based on Josephson junctions. It is a testament to the flexibility of the technology that such a broad array of quantum systems exist in it. The broadest two 

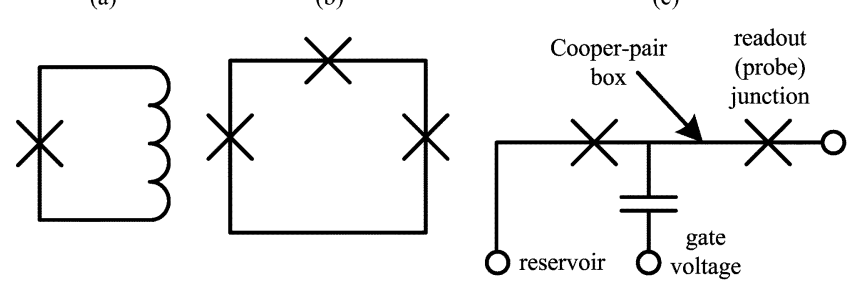

Fig. 4. (a) RF SQUID qubit, consisting of a single junction (cross) and an inductor. (b) Persistent-current qubit, consisting of three junctions in a SQUID loop. (c) Cooper-pair box qubit, consisting of an isolated metal island, and a readout mechanism (in the case shown here, a readout junction).

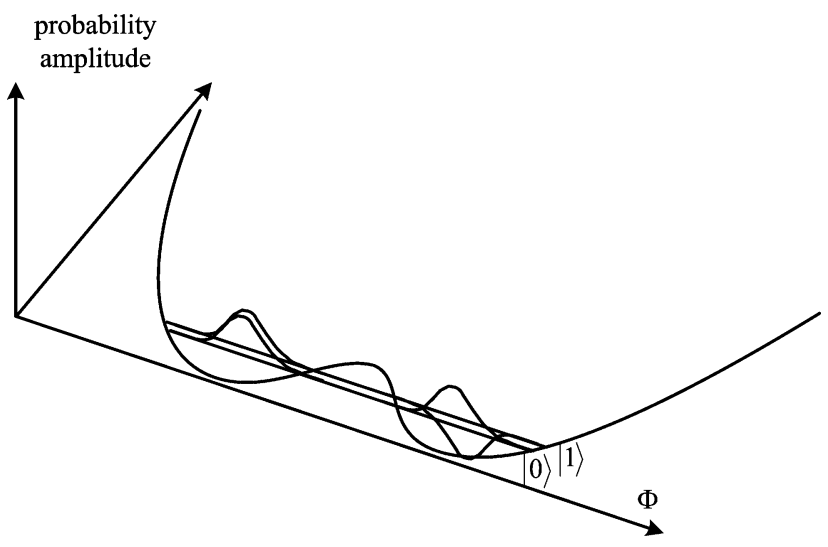

Fig. 5. Drawing of quantized states of a realistic potential for a superconductive qubit-the double well potential. This type of potential is formed in many of the superconductive qubit systems.

categories are defined by whether the quantum states in the qubit differ in the flux or in the charge stored in the junction. Here we speak of flux being "stored" very loosely-in fact the real physical quantity is a phase drop that exists in the wavefunction of the Cooper-pairs as it crosses the junction. Qubits differing in charge state are called "charge" qubits, those differing in phase state are called "phase" qubits. Typically in charge qubits, the uncertainty in the phase of the junctions is large, similarly in phase qubits, the uncertainty in the charge across the junctions is large.

1) Phase Qubits: Phase qubits subdivide further into a variety of subcategories. The easiest to describe consists of a single junction biased by a constant current [43]-[52]. The computational basis in this case is typically the lowest two energy states of a metastable local minimum of the potential energy of the junctions.

Another phase-based qubit, shown in Fig. 4(a), consists of a single junction enclosed in a loop, called an RF superconducting quantum interference device (SQUID) [53]-[56]. When an external field consisting of exactly half of a flux quantum is applied to the loop, the potential energy looks like a symmetric double well, shown in Fig. 5. The tunnel coupling between the wells permitted by the finite barrier height splits the ground-state degeneracy one would expect in the case of an infinite barrier. This splitting results in a symmetric ground state and antisymmetric first excited state for this system. To form a computational basis, we can take equally weighted positive and negative superpositions of these two states. The positive superposition has population in one well, the negative in the other. This choice of computational basis is not the only or even necessarily the best choice in this system, but it has the useful property that measurements of the flux stored in the loop can then reveal the computational state of the qubit.

A related phase-based qubit, the persistent-current qubit shown in Fig. 4(b), uses three junctions and has a very similar potential-energy diagram to that of the RF SQUID [57]-[65]. In this case, there are two degrees of freedom of the qubit, one of which can be neglected because the oscillation frequency in that direction is much higher than in the other. Along the axis of symmetry of the potential with the lower oscillation frequency, the system looks like a symmetric double well, just as in the RF SQUID qubit case. The choice of computational basis is the same as in the RF SQUID qubit case.

The final example of a phase-based qubit uses a very large Josephson junction, shaped by fabrication so that a single fluxon (a quantum of magnetic flux, which can also be thought of as a current vortex) moving in the junction sees a double-well potential [66]-[68]. Because fluxons are the fundamental particle of SFQ electronics (the classical logic family of superconductive electronics), this approach promises particularly smooth integration with classical control and readout electronics.

2) Charge Qubits: Charge-based qubits, shown in Fig. 4(c), all share the feature of using so-called Cooper-pair boxes as a key element [69]-[75]. These circuits consist of a small island of superconducting material (the Cooper-pair box) such that the capacitive charging energy of the island exceeds its thermal energy. A bias voltage on a nearby gate permits modulation of the box potential. When the box potential is set to the voltage corresponding to half a Cooper pair being on the box, the two states of the system (Cooper pair on and not on the box) are exactly degenerate and a symmetric double-well potential exists. The choice of computational basis is then the same as for the RF SQUID qubit, except now the double-well potential exists in the charge basis, not the phase basis.

3) Other Qubits: Qubits can also cross the boundary between the phase and charge categories. Proposals and experiments exist in which the quantum state of the system is defined in the charge basis, but the influence of this charge state on a coupled phase-sensitive circuit provides the qubit readout [76]-[79]. This approach eliminates undesirable voltage noise which is otherwise coupled to the qubit through the readout.

Finally, there are some proposals where no experimental implementation yet exists-in principle the $\pi$ phase drop inherent to high-temperature superconducting junctions could be used to simplify the problem of biasing the qubit [80]-[87]; however, no Josephson junctions have been made in these materials with anything close to the required quality for qubits. Also, there has been a proposal to use quantum bound states formed in the tunneling process (from so-called multiple Andreev reflections) to form a qubit [88]. 
Table 1

Truth Table for CNOT Gate

\begin{tabular}{c|c|c|c}
\hline \hline \multicolumn{2}{|c}{ Input } & \multicolumn{2}{c}{ Output } \\
\hline Control Bit & Target Bit & Control Bit & Target Bit \\
\hline 0 & 0 & 0 & 0 \\
\hline 0 & 1 & 0 & 1 \\
\hline 1 & 0 & 1 & 1 \\
\hline 1 & 1 & 1 & 0 \\
\hline \hline
\end{tabular}

The state of the target bit is flipped conditional on the state of the control bit.

\section{B. Single-Qubit Operations}

The "software" of a quantum computer is a sequence of classical instructions that execute one and two-bit operations on the computer. The driver circuits are classical circuits that take a digital input (instructions from the classical control system) and output an analog signal. Typically, one thinks of an RF pulse in the case of superconductive qubits [43], [63], [65], [77], [89] (but lower-frequency signals have also been used successfully [63], [69]), which then performs the single-qubit operation. The best classical analog of a singlequbit operation is the NOT operation in digital logic.

It is possible that control fields themselves can reveal the state of a qubit. For example, if a field with only a few photons is shone on a qubit, a subsequent measurement of the state of the field can reveal the state of the qubit [90], [91]. Straightforward calculations show that for superconductive qubits, if control fields with large numbers of photons are used, this effect can be safely neglected.

\section{Two-Qubit Operations}

Single-qubit operations are insufficient on their own to execute a quantum algorithm: some qubit couplings are required. Sequential action of two-qubit operations across the computer enable manipulation of superpositions of the states of multiple qubits: i.e., they generate entanglement in the quantum computer. The ability to generate entanglement is required in order to span the space of operations possible on a quantum computer.

The most elemental gate that can be used to generate entanglement in a quantum computer is the controlled-NOT (CNOT) gate. The truth table for this gate is shown in Table 1. This gate flips the target bit if and only if the control bit is on. To see why this seemingly innocuous gate can be used to generate entanglement, consider setting the input control bit to a superposition of zero and one $\left(\psi_{\text {control }}=\left(|0\rangle_{c}+|1\rangle_{c}\right) / \sqrt{2}\right)$. This can be done with a simple one-bit gate operation. The component of the control bit in the zero state will leave the target unchanged, while the component of the control in the one state will flip the state of the target. Then suppose the target starts out in the zero state $\left(\psi_{\text {target }}=|0\rangle_{t}\right)$, a measurement performed after the CNOT will always reveal one of two outcomes: the control and target bit are either both zero or both one $\left(\psi_{\text {final }}=\left(|0\rangle_{\mathrm{c}}|0\rangle_{\mathrm{t}}+|1\rangle_{\mathrm{c}}|1\rangle_{\mathrm{t}}\right) / \sqrt{2}\right)$. Because a measurement performed on one qubit now completely determines the state of the other (even though they are no longer interacting), we say they have become entangled.
The problem of implementing two-qubit gates like the CNOT gate is vastly simplified if controllable couplings (i.e., couplings whose strength can be modulated and ideally nulled to zero) are used. Aside from this constraint, virtually any physical interaction could be used as a coupler-in superconductive circuits, electromagnetic coupling with capacitors [48], [50], inductors [92]-[95], or microwave cavities [96], [97] have all been proposed, and a few have been recently realized experimentally [51], [75].

Work at the Massachusetts Institute of Technology (MIT) Lincoln Laboratory, Lexington, and the MIT campus, Cambridge, is aimed at developing a two-qubit coupler based on a balanced transformer design [94], [95]. The qubits in this case are coupled by two counterwound transformers whose inductance can be tuned with SQUIDs embedded in each transformer. This technique permits couplings that can be tuned to zero and then switched to a finite value on demand.

\section{Scaling $U p$}

Straightforward scaling of the results of [9] and [10] suggests that, depending on the choice of architecture, on the order of 1 million qubits will be required to factor a number large enough to be of practical interest. Superconductive circuits are interesting for application to quantum computing because they can be integrated using standard methods of very large scale integration [33], [34]. One challenge faced in scaling superconductive circuits will be control of device parameters. Like all approaches to quantum computing, each qubit is slightly different. In solid-state qubits, the dominant differences can be ascribed to fabrication imperfections, but in all approaches, variations in the local fields and coupling strengths to driver circuits will mandate qubit-by-qubit calibration of the control system. Because fabrication imperfections are likely to dominate the observed variance of superconductive qubit properties, development of methods for manufacturing qubits with nearly identical properties will be critical to practical implementation of a quantum computer.

An example of a fabrication process for superconductive quantum computing is one that has been implemented at MIT Lincoln Laboratory. The process is capable of yielding submicrometer Josephson junctions critical for the persistent-current qubit approach using i-line photolithography on 150-mm-diameter wafers [33]. Devices developed under this process have been used by multiple groups to realize superconductive quantum circuits [98], [99], [100].

\section{E. Sensor}

The sensor serves as the interface between the quantum world of the qubit and the classical world of the engineer, trying to extract useful (classical) information from the computer. At its core, the sensor consists of an amplifier and comparator. At some point in the measurement process, enough information about the signal has bled into the surrounding environment so that the experimenter could, at least in principle, detect it. At that point, the quantum information has been destroyed and only the classical information remains. The goal of a measurement circuit is then to amplify the 
signal while introducing a minimum of undesirable external action into the qubit.

Typically, the qubit in a superconductive quantum system has some dipole moment, either electric or magnetic, coupled either to a SQUID magnetometer or a single-electron transistor (SET) electrometer. Two kinds of coupling have been used successfully in qubit experiments.

1) "Always on" coupling, where the measurement is continuous, but weak, constantly reading out a value that is only loosely correlated to the value of the qubit. This method permits quantum operations to occur even while a measurement is being made but introduces unavoidable errors or decoherence.

2) Switchable coupling, where the measurement is turned on only at certain moments (once the quantum operations are complete). This method permits the qubit to be isolated from the outside world for the duration of the quantum operations, but then provides a classical measurement where the value is strongly correlated to the value of the qubit.

One measurement technique in development has several desirable features previously mentioned: it uses a symmetric superconductive comparator with a two-stage readout called a quantum flux parametron (QFP) [101], [102]. The symmetry of the device provides intrinsic rejection of low-frequency external noise. The two-stage readout separates the amplification stage from the classical measurement.

The jury is still out regarding measurement of superconductive qubits. The broad variety of methods that have emerged in a very short time, however, suggests that the measurement is unlikely to limit development of a superconductive quantum computer.

\section{F. Classical Control}

Unlike classical computers, quantum computers would not be used to write the programs that run on them. Instead, the sequence of gate operations that act on the qubits (the memory registers) to perform useful calculations can be thought of as the "program" or "software." The correct sequence has to be carried to and applied to the quantum computer using a classical computer — an analogy might be drawn to the days of the punch-card computer, where the punch card is created entirely separately from the computer. Additionally, the classical signals from the computer output have to be collected and brought out to the world. Of course, conventional silicon-based computers could be used here, but given the need for ultralow temperatures and large-scale calibration of the computer, it is likely that superconductive classical electronics would be a more convenient technology choice.

The most promising classical control electronics family (SFQ electronics) [38], [39] has been used extensively for implementation of analog, digital, and mixed-signal integrated circuits. Several proposals exist for use of these circuits to control quantum bit operations [40]-[42]. U1timately, however, a full-scale quantum computer would probably require a classical circuit much larger than has currently been achieved, and implementation of such a circuit is not easy. Additionally, a number of sources of noise, such as thermal [103] and electromagnetic [104], could be introduced in the process of integration of the SFQ circuits with the quantum device technology. Care will be needed to avoid these difficulties.

\section{REMAINING CHALLENGES}

There are two equally daunting challenges facing the field of superconductive quantum computing: decoherence and control. Both control inaccuracies and decoherence induce errors, which must be corrected in the computer. If these errors occur at a rate faster than some threshold [105], they cannot be corrected, and sustained computation is impossible.

Decoherence consists primarily of the effect that undesired interactions with the environment have on the computer, but also arises when the system entangles quantum mechanically with states of the qubit that are not part of the computational basis.

\section{A. Decoherence}

Without a demonstration of coherence times in excess of a microsecond, superconductive quantum computing is likely to fall flat on its face. Decoherence comes in two flavors: phase and amplitude. Phase decoherence, which is elastic in the sense that energy is not lost during the process, tends to be much faster than the inelastic amplitude decoherence, where energy is lost. The energy decay (or relaxation) times of typical superconductive qubits systems appear to be in the tens of microseconds, though the precise mechanisms of decay have not been identified [106]. Importantly, experiments have not been able to detect the very short time decoherence properties of the qubits, but this is the most critical timescale, because it is during the first few instants that the errors exceed the threshold required by the proposed error correction methods.

Recent experiments have shown the dephasing timescale of phase superconductive qubits to be $<100$ ns [43], [63], much smaller than the corresponding relaxation rates for these systems, which can exceed $20 \mu$ s [106]. To eliminate inhomogeneous broadening of the qubit levels due to low-frequency noise, spin-echo techniques borrowed from nuclear magnetic resonance technology must be used to measure the dephasing timescale.

The main sources of decoherence are still not completely understood. Typical models of the decoherence assume a coupling of a two-state quantum system-the qubit-to a bath of quantum-mechanical harmonic oscillators-the environment. The spectral density of the coupling to the bath is shaped by the frequency dependence of the electrical output impedance seen by the qubit [45], [47], [56], [60], [62], [64], [107]. It may still be, however, that other effects (for example, coupling to nuclear spins or charge states in the substrate or tunneling barriers) dominate the observed decoherence [107], [108]. 


\section{B. Control}

As with any system requiring analog control, systematic errors (caused, for example, by control electronics drifts or device parameter nonuniformity) build up rapidly. This feature makes the problem of control of a quantum computer much more challenging than control of a conventional computer, where the binary nature of the information can be used to reset the information at each stage and guard against buildup of errors. The theoretical solution to this problem (at the expense of greater system complexity) came with the discovery of quantum error correction [10], [105], which can reset the system as long as the total error rate does not exceed some threshold which depends somewhat on the type of error, but is typically estimated to be between 1 error per qubit per $10^{4}$ or $10^{5}$ operations.

But controlling an analog system consisting of many interacting qubits even with this level of precision is far from a trivial challenge. Achieving this control across an array of hundreds of thousands of qubits would an engineering tour de force.

\section{CONCLUSION}

Superconductive quantum computation faces many challenges before a computer large enough to be useful can be realized. However it has several key features that make it a promising approach to quantum computation.

1) It is a coherent quantum technology.

2) Superconductive devices can be fabricated and integrated monolithically.

3) Nearly ideal superconductive sensors exist.

4) A superconductive classical electronics family exists for device control.

These features suggest that this field is a strong competitor in the effort to develop a large-scale quantum computer.

The current government-sponsored road map for superconductive quantum computing calls for few-qubit systems to be developed in the next few years in order to understand the issues involved in implementation of the technology [109]. The field is well poised to achieve the goals laid out by this roadmap.

\section{ACKNOWLEDGMENT}

The author would like to thank Prof. T. Orlando, Dr. W. D. Oliver, Dr. P. Cho, Dr. L. You, Prof. D. Meyer, and Dr. J. Sage for helpful comments and discussions in preparing this paper.

\section{REFERENCES}

[1] P. W. Shor, "Algorithms for quantum computation: Discrete logarithms and factoring," presented at the 35th Annu. Symp. Foundations of Computer Science, Santa Fe, NM, 1994.

[2] M. E. Hellman, "An overview of public key cryptography," IEEE Commun. Soc. Mag., vol. 16, pp. 24-32, Nov. 1978.

[3] I. L. Chuang and M. A. Nielsen, Quantum Computation and Quantum Information. Cambridge, U.K.: Cambridge Univ. Press, 2000.

[4] A. Steane, "Quantum computing," Rep. Prog. Phys., vol. 61, pp. 117-173, 1998.
[5] D. V. Averin, "Quantum computing and quantum measurement with mesoscopic Josephson junctions," Fortschritte Phys., vol. 48, pp. 1055-1074, 2000.

[6] Y. Makhlin, G. Schon, and A. Shnirman, "Josephson-junction qubits," Fortschritte Phys., vol. 48, pp. 1043-1054, 2000.

[7] T. P. Spiller, "Superconducting circuits for quantum computing," Fortschritte Phys., vol. 48, pp. 1075-1094, 2000.

[8] Y. Makhlin, G. Schon, and A. Shnirman, "Quantum-state engineering with Josephson-junction devices," Rev. Mod. Phys., vol. 73, pp. 357-400, 2001.

[9] J. Cowie, B. Dodson, R. M. Elkenbracht-Huizing, A. K. Lenstra, P. L. Montgomery, and J. Zayer, "A world wide number field sieve factoring record: On to 512 bits," presented at the Advances in Cryptology Int. Conf. Theory and Applications of Cryptology and Information Security, Kyongju, South Korea, 1996.

[10] J. Preskill, "Reliable quantum computers," in Proc. R. Soc. London $A$, vol. 454, 1998, pp. 385-410.

[11] D. Beckman, A. N. Chari, S. Devabhaktuni, and J. Preskill, "Efficient networks for quantum factoring," Phys. Rev. A, vol. 54, pp. 1034-1063, 1996.

[12] L. M. K. Vandersypen, G. Breyta, M. Steffen, C. S. Yannoni, M. H. Sherwood, and I. L. Chuang, "Experimental realization of Shor's quantum factoring algorithm using nuclear magnetic resonance," Nature, vol. 414, pp. 883-887, 2001.

[13] C. Davisson and L. Germer, "The scattering of electrons by a single crystal of nickel," Nature, vol. 119, pp. 558-560, 1927.

[14] O. Nairz, M. Arndt, and A. Zeilinger, "Quantum interference experiments with large molecules," Amer. J. Phys., vol. 71, pp. 319-325, 2003.

[15] M. Arndt, O. Nairz, J. Petschinka, and A. Zeilinger, "High contrast interference with C/sub 60/ and C/sub 70/," Comptes Rendus de l'Academie des Sciences, Serie IV (Physique, Astrophysique), vol. 2, pp. 581-585, 2001.

[16] A. J. Leggett, "Macroscopic quantum systems and the quantum theory of measurement," Prog. Theor. Phys. Suppl., pp. 80-100, 1980.

[17] L. K. Grover, "A fast quantum mechanical algorithm for database search," presented at the 1996 ACM Symp. Theory of Computing, Philadelphia, PA, 1996.

[18] G. Brassard, "Searching a quantum phone book," Science, vol. 275, pp. 627-628, 1997.

[19] J. Yepez, "Quantum computation for physical modeling," Comput. Phys. Commun., vol. 146, pp. 277-279, 2002.

[20] - "Quantum lattice-gas model for the Burgers equation," J. Stat. Phys., vol. 107, pp. 203-224, Apr. 2002.

[21] , "Quantum lattice-gas model for the diffusion equation," Int. J. Mod. Phys. C, vol. 12, pp. 1285-1303, 2001.

[22] - "Type-II quantum computers," Int. J. Mod. Phys. C, vol. 12, pp. 1273-1284, 2001.

[23] ㄴ. "Quantum lattice-gas model for computational fluid dynamics," Phys. Rev. E, vol. 63, pp. 1-2, 2001.

[24] M. A. Pravia, C. Zhiying, J. Yepez, and D. G. Cory, "Toward a NMR implementation of a quantum lattice gas algorithm," Comput. Phys. Commun., vol. 146, pp. 339-344, 2002.

[25] G. P. Berman, A. A. Ezhov, D. I. Kamenev, and J. Yepez, "Simulation of the diffusion equation on a type-II quantum computer," Phys. Rev. A, vol. 66, pp. 1-8, 2002.

[26] J. P. Sage and K. K. Berggren, "Architecture for a type-II quantum computer coprocessor," MIT Lincoln Lab. Solid State Res. Q. Tech. Rep., vol. 1, pp. 37-42, 2003.

[27] E. Farhi, J. Goldstone, S. Gutmann, and M. Sipser. (2000) Quantum computation by adiabatic evolution. Quantum Phys. [Online]. Available: http://arxiv.org/pdf/quant-ph/0001106

[28] A. M. Childs, E. Farhi, and J. Preskill, "Robustness of adiabatic quantum computation," Phys. Rev. A, vol. 65, pp. 1-10, 2002.

[29] A. M. Childs, E. Farhi, J. Goldstone, and S. Gutmann. (2000) Finding cliques by quantum adiabatic evolution. Quantum Phys. [Online]. Available: http://arxiv.org/pdf/quant-ph/0012104

[30] W. M. Kaminsky and S. Lloyd, "Scalable architecture for adiabatic quantum computing of NP-hard problem," in Quantum Computing and Quantum Bits in Mesoscopic Systems. Norwell, MA: Kluwer, 2003.

[31] S. E. Venegas-Andraca and S. Bose, "Storing, processing and retrieving an image using quantum mechanics," presented at the Quantum Information and Computation Conf., Orlando, FL, 2003.

[32] D. A. Meyer and D. Curtis, "Toward quantum template matching," Proc. SPIE, Quantum Communications and Quantum Imaging, vol. 5161, pp. 134-141, 2003. 
[33] K. K. Berggren, E. M. Macedo, D. A. Feld, and J. P. Sage, "Low $\mathrm{T}_{c}$ /superconductive circuits fabricated on 150-mm-diameter wafers using a doubly planarized $\mathrm{Nb} / \mathrm{AlO}_{x} / \mathrm{Nb}$ process," IEEE Trans. Appl. Superconduct., vol. 9, pp. 3271-3274, June 1999.

[34] L. A. Abelson, Q. P. Herr, G. L. Kerber, M. Leung, and T. S. Tighe, "Manufacturability of superconductor electronics for a petaflops-scale computer," IEEE Trans. Appl. Superconduct., vol. 9, pp. 3202-3207, June 1999.

[35] J. Clarke, "Principles and applications of SQUIDs," Proc. IEEE, vol. 77, pp. 1208-1223, Aug. 1989.

[36] —-, "SQUIDs," Sci. Amer. (Int. Ed.), vol. 271, pp. 36-43, 1994.

[37] R. J. Schoelkopf, P. Wahlgren, A. A. Kozhevnikov, P. Delsing, and D. E. Prober, "The radio-frequency single-electron transistor (RFSET): A fast and ultrasensitive electrometer," Science, vol. 280, pp. $1238-1242,1998$.

[38] P. Bunyk, K. Likharev, and D. Zinoviev, "RSFQ technology: Physics and devices," Int. J. High Speed Electron. Syst., vol. 11, pp. 257-305, 2001.

[39] D. K. Brock, "RSFQ technology: Circuits and systems," Int. J. High Speed Electron. Syst., vol. 11, pp. 307-362, 2001.

[40] Z. Xingxiang, J. L. Habif, A. M. Herr, M. J. Feldman, and M. F. Bocko, "A tipping pulse scheme for a rf-SQUID qubit," IEEE Trans. Appl. Superconduct., vol. 11, pp. 1018-1021, Mar. 2001.

[41] R. C. R. de Castro, M. F. Bocko, A. M. Herr, C. A. Mancini, and M. J. Feldman, "Design of an RSFQ control circuit to observe MQC on an rf-SQUID," IEEE Trans. Appl. Superconduct., vol. 11, pp. 1014-1017, Mar. 2001.

[42] M. F. Bocko, A. M. Herr, and M. J. Feldman, "Prospects for quantum coherent computation using superconducting electronics," IEEE Trans. Appl. Superconduct., vol. 7, pp. 3638-3641, June 1997.

[43] J. M. Martinis, S. Nam, J. Aumentado, and C. Urbina, "Rabi oscillations in a large Josephson-junction qubit," Phys. Rev. Lett., vol. 89, pp. 117901-1-117901-4, 2002.

[44] Y. Yu, S. Han, X. Chu, S.-I. Chu, and Z. Wang, "Coherent temporal oscillations of macroscopic quantum states in a Josephson junction," Science, vol. 296, pp. 889-892, 2002.

[45] J. M. Martinis, S. Nam, J. Aumentado, K. M. Lang, and C. Urbina, "Decoherence of a superconducting qubit due to bias noise," Phys. Rev. B, Condens. Matter, vol. 67, pp. 94 510-1-94 510-10, 2003.

[46] P. R. Johnson, F. W. Strauch, A. J. Dragt, R. C. Ramos, C. J. Lobb, J. R. Anderson, and F. C. Wellstood, "Spectroscopy of capacitively coupled Josephson-junction qubits," Phys. Rev. B, Condens. Matter, vol. 67, pp. 20 509-1-20 509-4, 2003.

[47] A. J. Berkley, H. Xu, M. A. Gubrud, R. C. Ramos, J. R. Anderson, C. J. Lobb, and F. C. Wellstood, "Decoherence in a Josephson-junction qubit," Phys. Rev. B, Condens. Matter, vol. 68 , pp. 60 502-1-60502-4, 2003.

[48] R. C. Ramos, F. W. Strauch, P. R. Johnson, A. J. Berkley, H. Xu, M. A. Gubrud, J. R. Anderson, C. J. Lobb, A. J. Dragt, and F. C. Wellstood, "Capacitively coupled Josephson junctions: A two-qubit system," IEEE Trans. Appl. Superconduct., vol. 13, pp. 994-997, June 2003

[49] A. J. Berkley, H. Xu, M. A. Gubrud, R. C. Ramos, J. R. Anderson, C. J. Lobb, and F. C. Wellstood, "Characterization of an LC-isolated Josephson junction qubit," IEEE Trans. Appl. Superconduct., vol. 13, pp. 952-955, June 2003.

[50] F. W. Strauch, P. R. Johnson, A. J. Dragt, C. J. Lobb, J. R. Anderson, and F. C. Wellstood, "Quantum logic gates for coupled superconducting phase qubits," Phys. Rev. Lett., vol. 91, pp. $167005-$ $1-167005-4,2003$

[51] A. J. Berkley, H. Xu, R. C. Ramos, M. A. Gubrud, F. W. Strauch, P. R. Johnson, J. R. Anderson, A. J. Dragt, C. J. Lobb, and F. C. Wellstood, "Entangled macroscopic quantum states in two superconducting qubits," Science, vol. 300, pp. 1548-1550, 2003.

[52] A. Wallraff, T. Duty, A. Lukashenko, and A. V. Ustinov, "Multiphoton transitions between energy levels in a current-biased Josephson tunnel junction," Phys. Rev. Lett., vol. 90, pp. 037 003-1-037 003-4, 2003.

[53] R. Rouse, S. Han, and J. E. Lukens, "Resonant tunneling between macroscopically distinct levels of a SQUID," in Proc. 2nd Int. Conf. Phenomenology of Unification from Present to Future, 1998, pp. 207-224.

[54] J. R. Friedman, V. Patel, W. Chen, S. K. Tolpygo, and J. E. Lukens, "Quantum superposition of distinct macroscopic states," Nature, vol. 406, pp. 43-46, 2000.

[55] S. Han, R. Rouse, and J. E. Lukens, "Observation of cascaded two-photon-induced transitions between fluxoid states of a SQUID," Phys. Rev. Lett., vol. 84, pp. 1300-1303, 2000.
[56] D. V. Averin, J. R. Friedman, and J. E. Lukens, "Macroscopic resonant tunneling of magnetic flux," Phys. Rev. B, Condens. Matter, vol. 62, pp. $11802-11811,2000$.

[57] J. E. Mooij, T. P. Orlando, L. Levitov, T. Lin, C. H. van der Wal, and S. Lloyd, "Josephson persistent-current qubit," Science, vol. 285, pp. 1036-1039, 1999.

[58] T. P. Orlando, J. E. Mooij, L. Tian, C. H. van der Wal, L. S. Levitov, S. Lloyd, and J. J. Mazo, "Superconducting persistent-current qubit," Phys. Rev. B, Condens. Matter, vol. 60, pp. 15 398-15413, 1999.

[59] C. H. van der Wal, A. C. J. Ter-Haar, F. K. Wilhelm, R. N. Schouten, C. J. P. M. Harmans, T. P. Orlando, S. Lloyd, and J. E. Mooij, "Quantum superposition of macroscopic persistent-current states," Science, vol. 290, pp. 773-777, 2000.

[60] L. Tian, L. S. Levitov, C. H. van der Wal, J. E. Mooij, T. P. Orlando, S. Lloyd, C. J. P. M. Harmans, and J. J. Mazo, "Decoherence of the superconducting persistent current qubit," presented at the NATO Advanced Study Inst. Quantum Mesoscopic Phenomena and Mesoscopic Devices in Microelectronics, Ankara, Turkey, 2000.

[61] T. P. Orlando, S. Lloyd, L. S. Levitov, K. K. Berggren, M. J. Feldman, M. F. Bocko, J. E. Mooij, C. J. P. Harmans, and C. H. van der Wal, "Flux-based superconducting qubits for quantum computation," Physica C, vol. 1, pp. 194-200, 2002.

[62] T. P. Orlando, L. Tian, D. S. Crankshaw, S. Lloyd, C. H. van der Wal, J.E. Mooij, and F. Wilhelm, "Engineering the quantum measurement process for the persistent current qubit," Physica $C$, vol. 368, pp. 294-299, 2002.

[63] I. Chiorescu, Y. Nakamura, C. J. P. M. Harmans, and J. E. Mooij, "Coherent quantum dynamics of a superconducting flux qubit," Science, vol. 299, pp. 1869-1871, 2003.

[64] C. H. van der Wal, F. K. Wilhelm, C. J. P. M. Harmans, and J. E. Mooij, "Engineering decoherence in Josephson persistent-current qubits. Measurement apparatus and other electromagnetic environments," Eur. Phys. J. B, vol. 31, pp. 111-124, 2003.

[65] Y. Nakamura, I. Chiorescu, H. Mooij, and C. J. P. M. Harmans, "Josephson-junction flux qubit," NEC Res. Develop., vol. 44, pp. 278-281, 2003.

[66] A. Kemp, A. Wallraff, and A. V. Ustinov, "Josephson vortex qubit: Design, preparation, and read-out," Physica Status Solidi B, vol. 233, pp. $472-481,2002$.

[67] - "Testing a state preparation and read-out protocol for the vortex qubit," Physica C, vol. 368, pp. 324-327, 2002.

[68] A. V. Ustinov, A. Walraff, and Y. Koval, "Shaped Josephson junction qubits," U. S. Patent 6627915, Aug. 11, 2000

[69] Y. Nakamura, Y. A. Pashkin, and J. S. Tsai, "Coherent control of macroscopic quantum states in a single-Cooper-pair box," Nature, vol. 398, pp. 786-788, 1999.

[70] Y. Nakamura and J. S. Tsai, "Josephson-junction quantum bits," Oyo Buturi, vol. 69, pp. 1299-1304, 2000.

[71] J. S. Tsai, Y. Nakamura, and Y. Pashkin, "Qubit utilizing charge-number state in superconducting nanostructure," Quantum Inf. Comput., vol. 1, pp. 124-128, 2001.

[72] - "Superconducting single-Cooper-pair box as quantum bit," Physica C, vol. 357, pp. 1-6, 2001.

[73] - "Superconducting single-Cooper-pair box quantum bit with multi-gate-pulse operation,” Physica C, vol. 367, pp. 191-196, 2002.

[74] Y. A. Pashkin, T. Yamamoto, O. Astafiev, Y. Nakamura, D. V. Averin, and J. S. Tsai, "Quantum oscillations in two coupled charge qubits," Nature, vol. 421, pp. 823-826, 2003.

[75] Y. Pashkin, T. Yamamoto, O. Astafiev, Y. Nakamura, D. Averin, and J.-S. Tsai, "Coupling two charge qubits," NEC Res. Develop., vol. 44, pp. 273-277, 2003.

[76] J. R. Friedman and D. V. Averin, "Aharonov-Casher-effect suppression of macroscopic tunneling of magnetic flux," Phys. Rev. Lett., vol. 88, pp. 050 403-1-050 403-4, 2002.

[77] A. Cottet, D. Vion, A. Aassime, P. Joyez, D. Esteve, and M. H. Devoret, "Implementation of a combined charge-phase quantum bit in a superconducting circuit," Physica C, vol. 367, pp. 197-203, 2002.

[78] D. Vion, A. Aassime, A. Cottet, P. Joyez, H. Pothier, M. H. Devoret, C. Urbina, and D. Esteve, "Toward quantum electrical circuits," Physica E, vol. 18, pp. 7-10, 2003.

[79] D. Vion, A. Aassime, A. Cottet, P. Joyez, H. Pothier, C. Urbina, D. Esteve, and M. H. Devorett, "Manipulating the quantum state of an electrical circuit," Science, vol. 296, pp. 886-889, 2002.

[80] A. Blais and A. M. Zagoskin, "Operation of universal gates in a solid-state quantum computer based on clean Josephson junctions between d-wave superconductors," Phys. Rev. A, Gen. Phys., vol. 61, pp. 042 308-1-042 308-4, 2000. 
[81] A. M. Zagoskin, "d-Wave superconductors and quantum computers," Physica C, vol. 368, pp. 305-309, 2002.

[82] M. H. S. Amin, A. N. Omelyanchouk, A. Blais, A. M. van den Brink, G. Rose, T. Duty, and A. M. Zagoskin, "Multi-terminal superconducting phase qubit," Physica C, vol. 368, pp. 310-314, 2002.

[83] A. M. Zagoskin, "Qubit using a Josephson junction between s-wave and d-wave superconductors," U.S. Patent 6459 097, Jan. 7, 2000.

[84] A. Y. Tzalenchuk, T. Lindstrom, S. A. Charlebois, E. A. Stepantsov, A. M. Zagoskin, Z. Ivanov, and T. Claeson, "Feasibility studies of ultra-small Josephson junctions for qubits," IEEE Trans. Appl. Superconduct., vol. 13, pp. 948-951, June 2003.

[85] —, "Quantum computing method using magnetic flux states at a Josephson junction," U.S. Patent 6563311, May 14, 2001.

[86] A. M. Zagoskin, "Quantum computing method using Josephson junctions between s-wave and d-wave superconductors," U.S. Patent 6563310, May 14, 2001.

[87] A. M. Zagoskin, G. Rose, M. H. S. Amin, M. Franz, and J. P. Hilton, "Superconducting dot/anti-dot flux qubit based on time-reversal symmetry breaking effects," U.S. Patent 6537 847, Jan. 25, 2002.

[88] S. E. Shafranjuk, I. P. Nevirkovets, and J. B. Ketterson, "A qubit device based on manipulations of Andreev bound states in doublebarrier Josephson junctions," Solid State Commun., vol. 121, pp. 457-460, 2002.

[89] S. Han, Y. Yu, X. Chu, S.-I. Chu, and Z. Wang, "Time-resolved measurement of dissipation-induced decoherence in a Josephson junction," Science, vol. 293, pp. 1457-1459, 2001.

[90] S. J. van Enk and H. J. Kimble, "Strongly focused light beams interacting with single atoms in free space," Phys. Rev. A, vol. 63, pp. 023 809-1-023 809-9, 2001.

[91] — - "On the classical character of control fields in quantum information processing," Quantum Inf. Comput., vol. 2, pp. 1-13, 2002.

[92] J. E. Mooij, T. P. Orlando, L. Levitov, L. Tian, C. H. van der Wal, and S. Lloyd, "Josephson persistent-current qubit," Science, vol. 285, pp. 1036-1039, 1999.

[93] M. J. Storcz and F. K. Wilhelm, "Design of realistic switches for coupling superconducting solid-state qubits," Appl. Phys. Lett., vol. 83, pp. 2387-2389, 2003.

[94] T. V. Filippov, S. K. Tolpygo, J. Mannik, and J. E. Lukens, "Tunable transformer for qubits based on flux states," IEEE Trans. Appl. Superconduct., vol. 13, pp. 1005-1008, June 2003.

[95] J. P. Sage, K. K. Berggren, and W. D. Oliver, "Design of a coupled two-qubit quantum computer element," MIT Lincoln Lab. Solid State Res. Rep., no. 3, pp. 37-42, 2003.

[96] C.-P. Yang, S.-I. Chu, and S. Han, "Possible realization of entanglement, logical gates, and quantum-information transfer with superconducting-quantum-interference-device qubits in cavity QED," Phys. Rev. A, Gen. Phys., vol. 67, pp. 42 311-1-42 311-8, 2003.

[97] M. V. Fistul and A. V. Ustinov, "Josephson vortex interaction mediated by cavity modes: Tunable coupling for superconducting qubits," Phys. Rev. B, Condens. Matter, vol. 68, pp. 132 509-1-132 509-4, 2003.

[98] R. Koch, private communication, 2003.

[99] K. Segall, D. S. Crankshaw, D. Nakada, B. Singh, J. Lee, T. P. Orlando, K. K. Berggren, N. Markovic, S. O. Valenzuela, and M. Tinkham, "Experimental characterization of the two current states in a $\mathrm{Nb}$ persistent-current qubit," IEEE Trans. Appl. Superconduct., vol. 13, pp. 1009-1012, June 2003.

[100] K. Segall, D. Crankshaw, D. Nakada, T. P. Orlando, L. S. Levitov, S. Lloyd, N. Markovic, S. O. Valenzuela, M. Tinkham, and K. K. Berggren, "Impact of time-ordered measurements of the two states in a niobium superconducting qubit structure," Phys. Rev. B, Condens. Matter, vol. 67, pp. 220 506-1-220 506-4, 2003.
[101] D. A. Feld, J. P. Sage, K. K. Berggren, and A. Siddiqui, "Measurement of the energy sensitivity of a superconductive comparator," IEEE Trans. Appl. Superconduct., vol. 9, pp. 4361-4366, June 1999.

[102] W. D. Oliver, K. K. Berggren, J. P. Sage, T. P. Orlando, T. J. Weir, R. L. Slattery, and G. Fitch, "Quantum flux parametron as a readout element for a persistent-current qubit," MIT Lincoln Lab. Solid State Res. Rep., no. 2, 2003.

[103] T. A. Ohki, J. L. Habif, M. J. Feldman, and M. F. Bocko, "Thermal design of superconducting digital circuits for millikelvin operation," IEEE Trans. Appl. Superconduct., vol. 13, pp. 978-981, June 2003.

[104] J. L. Habif and M. F. Bocko. (2003) Decoherence of the flux-based superconducting qubit in an integrated circuit environment. Quantum Phys. [Online]. Available: http://arxiv.org/pdf/quant-ph/ 0304045

[105] P. W. Shor, "Fault-tolerant quantum computation," presented at the 37th Conf. Foundations of Computer Science, Burlington, VT, 1996.

[106] Y. Yu, D. Nakada, J. C. Lee, B. Singh, D. S. Crankshaw, T. P. Orlando, K. K. Berggren, and W. D. Oliver, "Energy relaxation time between macroscopic quantum levels in a superconducting persistentcurrent qubit," Phys. Rev. Lett., vol. 92, pp. 117904-4-117904-4, 2004.

[107] E. Paladino, L. Faoro, G. Falci, and R. Fazio, "Decoherence and $1 / f$ noise in Josephson qubits," Phys. Rev. Lett., vol. 88, pp. $228304-$ 1-228 304-4, 2002.

[108] M. Dube and P. C. E. Stamp, "Mechanisms of decoherence at low temperatures," Chem. Phys., vol. 268, pp. 257-272, 2001.

[109] Superconducting approaches to quantum information processing and quantum computing: A quantum information science and technology roadmap-Part 1: Quantum computation (2004). [Online]. Available: http://qist.lanl.gov/pdfs/supercond.pdf

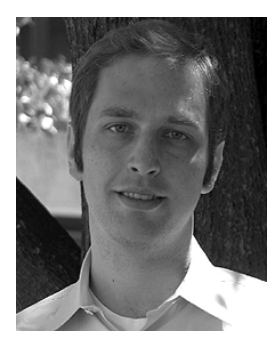

Karl K. Berggren (Member, IEEE) was born in New Westminster, B.C., Canada, on August 20, 1970. He received the B.A. degree (cum laude) and the Ph.D. degree in physics from Harvard, Cambridge, MA, in 1990 and 1997, respectively. His thesis work focused on nanolithographic methods using neutral atoms.

From 1996 to 2003, he was a Staff Member at the Massachusetts Institute of Technology Lincoln Laboratory, Lexington. He is currently an Assistant Professor of Electrical Engineering, Department of Electrical Engineering and Computer Science, Massachusetts Institute of Technology, Cambridge, where he heads the Quantum Nanostructures and Nanofabrication group. He is also Associate Director of the Nanostructures Laboratory in the Research Laboratory of Electronics. His research focuses on methods of nanofabrication, especially applied to superconductive quantum circuits, photodetectors, and high-speed superconductive electronics.

Prof. Berggren is a member of the American Physical Society. He has also served on the program committee for the Applied Superconductivity Conference. 